\title{
La chute du censeur dans le cinéma polonais (Wojciech Marczewski et Krzysztof Zanussi)
}

\section{Tadeusz Szcepański}

Traducteur : Alice Pisarek

\section{OpenEdition \\ Journals}

Édition électronique

URL : http://journals.openedition.org/recherchestravaux/512

DOI : 10.4000/recherchestravaux.512

ISSN : 1969-6434

Éditeur

UGA Éditions/Université Grenoble Alpes

Édition imprimée

Date de publication : 15 mai 2012

Pagination : 75-85

ISBN : 978-2-84310-227-1

ISSN : 0151-1874

\section{Référence électronique}

Tadeusz Szcepański, «La chute du censeur dans le cinéma polonais (Wojciech Marczewski et Krzysztof Zanussi) », Recherches \& Travaux [En ligne], 80 | 2012, mis en ligne le 15 novembre 2013, consulté le 08 septembre 2020. URL : http://journals.openedition.org/recherchestravaux/512 ; DOI https://doi.org/10.4000/recherchestravaux.512 


\section{La chute du censeur dans le cinéma polonais (Wojciech Marczewski et Krzysztof Zanussi)}

Durant la période charnière de l'Histoire contemporaine de la Pologne, qui, en 1989, a changé de régime politique et a retrouvé son indépendance en se libérant du joug politique d'un empire soviétique en déclin, deux films sont sortis sur les écrans dont les personnages principaux étaient des censeurs : L'Inventaire (Stan posiadania, 1989) ${ }^{\mathrm{I}}$ de Krzysztof Zanussi et L'Évasion du cinéma "Liberté» (Ucieczka z kina "Wolność", 1990) de Wojciech Marczewski. Les deux films abordaient la condition morale et psychique des fonctionnaires de la censure, dont la tâche consistait à éliminer des livres, journaux, films, spectacles, etc., avant leur diffusion publique, les informations et opinions indésirables du point de vue de l'idéologie et de la politique en vigueur, et à promouvoir les valeurs conformes à la propagande communiste. La censure politique en Pologne qui, après la Seconde Guerre mondiale, s'est trouvée dans l'orbite de l'Union soviétique, était un pilier du système totalitaire. Elle bloquait de façon efficace les aspirations démocratiques de la société et toutes les tentatives de reconquérir la liberté et l'indépendance. Un des fonctionnaires de la censure, Tomasz Strzyżewski, est parvenu à démasquer son fonctionnement. En 1977, il a transporté clandestinement vers la Suède plusieurs documents secrets contenant des instructions destinées aux censeurs. La publication de ces textes par l'éditeur de l'émigration polonaise de Londres Aneks ${ }^{2}$ a révélé l'ampleur des dégâts de la censure et a conduit les

I. Le film a été projeté en France sous le titre L'Inventaire. La traduction littérale du titre original serait : «L'état des possessions».

2. T. Strzyżewski, Czarna Ksiega cenzury PRL (Le Livre noir de la censure PRL), Londres, Aneks, 1977. 
ouvriers des chantiers navals de Gdańsk à inclure la liberté d'expression dans leurs vingt et une revendications lors de la grève d'août 198I, qui a donné naissance au syndicat Solidarność.

De tous les modes d'expression, c'est le cinéma qui a été le plus exposé à la censure. "Le plus important de tous les arts", selon la célèbre formule de Lénine, en raison de sa portée sociale, a subi un contrôle extrêmement rigoureux et scrupuleux. Dès 1945, date de la nationalisation du cinéma polonais, les cas d'intrusion de la censure, tant préventive que répressive, se sont multipliés. À toutes les étapes de la création, depuis l'écriture du scénario jusqu'à l'achèvement de l'œuvre, les films étaient obligatoirement soumis à l'acceptation de l'institution adéquate. Après l'instauration de l'état de siège, le 13 décembre 1981, le Bureau général du contrôle de la presse, des publications et des spectacles (Główny Urzad Kontroli Prasy Publikacji i Widowisk) - tel était le nom officiel de cette institution - a fait des dégâts particulièrement sévères et douloureux dans le domaine du cinéma. Une série de films de grande qualité, tournés pendant les années 1980-I98I, c’est-àdire au moment où la censure a été obligée d'assouplir ses critères d'évaluation politique sous la pression d'une société révoltée, s'est retrouvée sur la liste noire. Cette liste comprenait des films portant un regard critique sur la société contemporaine, comme Le Hasard de Krzysztof Kieślowski et Une femme seule d'Agnieszka Holland. Cependant, la censure a plus durement encore touché les films qui dénonçaient les travers politiques et la terreur du régime stalinien. Ces œuvres sont nées de la brèche, courageusement ouverte dans un mutisme généralisé, par Andrzej Wajda avec son film L'Homme de marbre ${ }^{3}$ en 1976. À la faveur du mouvement de libéralisation lancé par Solidarność, d'autres jeunes réalisateurs, nés dans les années I940 et dont l'enfance s'est déroulée sous le stalinisme, ont suivi ses traces. On mentionnera tout particulièrement : Matka Królów (La Mère des rois) de Janusz Zaorski, Wahadetko (La Pendulette) de Filip Bajon, Niech cię odleci mara (Que ce fantôme disparaisse) d'Andrzej Barański, Wielki Bieg (La Grande Course) de Jerzy Domaradzki, Dreszcze (Frissons) de Wojciech Marczewski, ainsi que Przestuchanie (L'Interrogatoire) de Ryszard Bugajski. Ce dernier film fera l'objet d'un acharnement obstiné de la part du pouvoir pendant l'état de siège. La réalisation de ces films dans un contexte de révolte antitotalitaire peut être perçue comme un symptôme du démantèlement de l'Union soviétique. En effet, le stalinisme, dénoncé lors du $\mathrm{XX}^{\mathrm{e}}$ congrès du PCUS, est rapidement réapparu sous la forme édulcorée du régime néostalinien instauré par

3. Ceci sans compter Haut les mains de J. Skolimowski, film censuré, ce qui a décidé le réalisateur à émigrer. 
Léonid Brejnev. Ainsi, après le I3 décembre 198I, lorsque le pouvoir a voulu reprendre le contrôle sur la population polonaise au moyen de la force, les films qui avaient osé mettre en scène la domination de l'État sur l'individu ont été éliminés de l'espace public. Et la censure, parce qu'elle a intensifié ses agissements au moment de l'état de siège, est devenue l'ennemi public numéro un dans le milieu cinématographique.

Pourtant, les films mettant en scène des fonctionnaires de la censure, tournés au moment de la chute de la République populaire de Pologne (L'Inventaire) ou bien juste après (L'Évasion du cinéma "Liberté»), ne revêtent pas le caractère d'une vengeance pour les torts et les humiliations subies. Ils se rapprochent plutôt du genre de la moralité et s'efforcent de mettre en avant et de comprendre les mobiles des censeurs, représentés comme des êtres déchus et frustrés. Ces derniers se sont simplement perdus "sur les sentiers où l'herbe repousse" - pour reprendre le titre du dernier ouvrage de Knut Hamsun -, sont tombés dans les pièges tendus par la vie, mais grâce à l'aide de leurs proches, ils peuvent en sortir et réparer leurs torts passés (même s'ils n'y arrivent pas toujours). Dans L'Inventaire comme dans L'Évasion $d u$ cinéma "Liberté", apparaissent des personnages protecteurs qui tendent la main aux fonctionnaires de la censure et s'efforcent de les ramener du côté lumineux de l'existence. Mais si les deux réalisateurs ont un regard chargé d'empathie envers leurs personnages dévoyés, ils n'en livrent pas moins un témoignage éthique sévère sur leurs comportements. Dans les deux films, les censeurs sont des êtres immoraux et corrompus par le pouvoir, parce qu'ils ont succombé à la tentation des privilèges et de l'argent. Ils ont cependant des sursauts, des prises de conscience de leur immoralité, mais à différents degrés. Enfin, l'action de ces deux films se déroule dans la deuxième moitié des années I980, au moment de la décomposition du régime communiste en Pologne et à la veille de sa chute finale, ce qui confere aux péripéties des héros une dimension symbolique, voire allégorique.

\section{L'Inventaire de Krzysztof Zanussi}

Dans L'Inventaire, Krzysztof Zanussi nous livre l'histoire d'une femme déchue qui, du jour au lendemain, perd tous les moyens matériels de son existence. Elle perd son travail, doit quitter son appartement et finit à la rue. Ne pouvant compter ni sur ses amis ni sur sa famille, Julia frôle la catastrophe, mais y échappe grâce à l'intérêt que lui manifeste, de façon inespérée, un étudiant, intrigué par ses comportements névrotiques. Il l'accueille chez lui, prend soin d'elle et s'efforce de comprendre, pas à pas, les raisons de sa lamentable 
situation. Tomek est un garçon élevé dans la foi chrétienne par une mère qui lui a appris qu'il fallait toujours aider son prochain en détresse. Il emmène Julia dans l'appartement qu'il habite avec sa mère, lui propose du réconfort et un toit. Son comportement altruiste, qui se transforme rapidement en un sentiment, réciproque, à l'égard d'une femme plus âgée que lui d'une bonne dizaine d'années, confronte sa mère à une rude épreuve morale. Julia est, en effet, l'exact opposé de la femme idéale dont la mère, très religieuse, rêvait pour son fils unique chéri. Abandonnée par son mari, cette dernière a élevé seule son enfant, en lui inculquant la morale chrétienne. Julia, elle, fait preuve d'une totale indifférence à l'égard de la religion. Elle semble vivre au jour le jour, sombrant, sans foi ni loi, dans le chaos de la vie. Son parler vulgaire et son attitude révèlent un manque d'éducation et ses habits clinquants parachèvent le tableau. Tomek a rencontré Julia dans une clinique. Abandonnée par son amant, elle vient de subir un avortement. Cet acte inadmissible du point de vue de la morale catholique suscite une sévère réprobation de la part du jeune homme. On devine que la fin orageuse de la romance de Julia est la cause de son licenciement et que son partenaire est un collègue censeur hiérarchiquement supérieur. Le dialogue entre Zofia, la mère de Tomek, et Julia, au cours duquel la jeune femme révèle son ancien métier, permet d'accentuer la caractérisation négative de l'ex-censeur(e). Son aveu constitue pour Zofia un choc d'autant plus retentissant qu'elle et son fils partagent le combat mené par Solidarność. Tomek a même colporté les ouvrages du «second circuit» (Samizdat), imprimés et diffusés hors du contrôle de la censure. Il vaut la peine de citer ici un long fragment du dialogue en question car il livre une image juste de la mentalité ambivalente de la fonctionnaire de la censure. Lorsque Zofia, souhaitant aider Julia, cherche à en savoir davantage sur elle, Julia dévoile, non sans une certaine gêne, son métier :

Julia :... Je n'en parle pas facilement... Je travaillais dans la censure. Je savais bien que ça n'allait pas vous plaire, dans tous les cas ça ne vous laissera pas indifférente...

Zofia : Je ne vois pas comment ça pourrait me plaire... C'est précisément parce qu'il y a une censure, qu'il a un système clandestin. Tomek, mon fils, colporte les ouvrages clandestins. Voilà que je suis amenée à rencontrer une personne qui... J'aimerais comprendre.... Comment vous... Vous travaillez dans la censure de la presse, des journaux?

Julia : Oui... Je ne travaille pas, je travaillais, mais c'est sans importance...

Zofia (prenant un journal) : Par exemple, vous avez là un article [...], là c'est censuré... [...] Comment vous décidez de supprimer telle ou telle chose?

Julia : J'ai des instructions à suivre tout simplement. Je sais ce qu'on ne peut pas imprimer. Je lis et j'évalue de ce point de vue. [...] Oui, on sait qu'il ne faut pas laisser imprimer le nom de certaines institutions ou certains faits, il y a des listes de noms, on me les donne et je les élimine [...] je ne fais que mon travail, 
je gagne ma vie de cette façon. Je ne me pose pas de questions, en tout cas je ne m'en posais pas...

Zofia : Mais vous censurez ce que quelqu'un d'autre a dit et non pas ce vous pensez...

Julia : Si chaque censeur devait mettre son nom en bas de l'article, ce serait différent. Moi, je ne signe pas de mon nom, j'exécute des instructions et c'est tout. [...] Non, je ne me posais pas de questions, la plupart de mes collègues non plus. Et puis on ne rencontre jamais ces gens, si c'était le cas, ce serait différent. [...] Le plus souvent, on élimine de telle façon que vous ne vous rendez même pas compte que quelque chose a été éliminé. Un bon censeur fait en sorte que personne ne se rende compte.

Zofia : Je ne serais pas un bon censeur, alors...

Julia : Moi j'en étais un.

Dévoilant les rouages de ce métier avilissant, qui consistait à falsifier habilement et à effacer les traces de la moindre critique sociale, Julia s'efforce de se décharger de toute responsabilité morale dans l'exercice de son métier. Elle semble certes consciente que ses agissements étaient honteux et répréhensibles. Mais elle tente de neutraliser tout jugement moral sur cette activité en suggérant que c'était un travail comme un autre, une simple source de revenus. En expliquant qu'elle a agi conformément aux exigences de son métier, elle se libère du sentiment de culpabilité et échappe aux accusations. Censeur anonyme et simple exécutant, elle n'a fait qu'appliquer des consignes. Sa ligne de défense, toutes proportions gardées, rappelle les arguments des criminels de guerre qui essayaient de justifier leurs crimes en invoquant les ordres de leurs supérieurs. On peut également penser que son engagement du côté du pouvoir était bien plus profond encore. C'est ce que suggère la scène dans le bureau qui délivre les passeports, où Julia tente d'obtenir un passeport grâce à ses relations, en rappelant d'autres services, plus honteux encore, qu' elle a rendus autrefois.

Dans L'Inventaire, Krzysztof Zanussi confronte deux postures très contrastées : Julia est dépourvue de tout principe moral, adepte irréfléchie du régime communiste appelé à disparaitre, parce qu'il s'appuyait sur la faiblesse, l'esclavage et la corruption; Zofia et son fils sont des êtres purs, qui permettent d'espérer un avenir véritablement «radieux». Même si le réalisateur complique ce dispositif manichéen en soumettant la foi chrétienne de Zofia à l'épreuve de l'acceptation de la pécheresse Julia, le mal est en définitive vaincu. Plongée dans le chaos moral, la malheureuse Julia perd la raison et finit dans un hôpital psychiatrique. Tomek et Zofia, en revanche, éprouvent, une fois de plus, le pouvoir salvateur du bien et sont à la fin du film plus que jamais convaincus d'avoir choisi la bonne voie.

Lors de sa sortie en salles, le film de Krzysztof Zanussi a obtenu des critiques contrastées : le public polonais lui a fait un accueil mitigé, mais le film 
a été remarqué et primé lors de festivals à l'étranger (en France, il a obtenu, en 1990, le grand prix du Festival de Strasbourg organisé par l'Institut des Droits de l'Homme). L'Évasion du cinéma "Liberté», en revanche, passe pour l'un des meilleurs films polonais contemporains. Il a été récompensé par de nombreux prix, notamment par le Lion d'or, la plus haute distinction au Festival du film polonais de Gdynia.

\section{L'Évasion du cinéma «Liberté» de Wojciech Marczewski}

Dans L'Inventaire, film intimiste, le métier de censeur est uniquement un trait complémentaire du caractère négatif de l'un des trois personnages principaux. Il ne constitue qu'un des aspects les plus destructeurs de sa personnalité dégradée, fruit de l'idéologie nihiliste d'un régime en déclin. A contrario, dans L'Évasion du cinéma "Liberté» de Wojciech Marczewski, le métier de censeur définit et caractérise presque entièrement le personnage principal. De plus, si Julia était simplement un élément mineur de la machine de la censure et se contentait d'exécuter mécaniquement les instructions de ses supérieurs, le censeur du film de Wojciech Marczewski se place à un échelon nettement plus élevé de la hiérarchie. Comme responsable de la censure de toute une région il a déjà à son actif quinze longues années d'expérience dans l'institution et, bien qu'il soit un individu cynique, parfaitement conscient de l'ignominie de ses activités ("Il a trempé ses mains dans plus d'une merde», dit-il en parlant de lui-même), il s'efforce d'anoblir l'art de la censure. Dans la première scène du film, il forme des rédacteurs en chef de différents médias et légitime son métier en exposant son credo professionnel :

La censure est nécessaire. Censurer est un art. Un bon censeur est un artiste. Mais c'est aussi un jeu. Par exemple, si quelqu'un écrit "À bas le communisme», on sait immédiatement qu'il faut intervenir... mais si quelqu'un écrit "cochon", il faut se demander si l'auteur parle d'une ferme ou du pouvoir. Laissons pour l'instant de côté les banalités sur l'inquisition et la démocratie. Je ne sais pas si vous êtes conscients qu'au moment de la liquidation de la censure en tant qu'institution, c'est sur vous que retombera le devoir de censurer ${ }^{4}$.

4. Ce discours se rapporte à des faits réels. Dans les années 1970, un censeur a été licencié après avoir autorisé la légende "En haut, le bétail se nourrit» sous la photo d'un troupeau de moutons broutant sur une colline (B. Janicka, "Czyściec cenzora» "Le purgatoire du censeur»], Film, n 35, 1990. [NdT : en polonais le mot "bétail» signifie également «salaud».] Dans le même temps, est née l'idée d'abolir la censure et de confier cette tâche aux directeurs des médias et des sociétés de production cinématographiques. Le projet s'est heurté à une vive protestation de la part des milieux artistiques (voir la déclaration de K. Zanussi, dans le documentaire de K. Magowski intitulé «Tren na śmierć cenzora» ["Thrène sur la mort du censeur»] réalisé en 1992). 
Malgré son assurance apparente, due à plusieurs années de familiarité avec les organes du pouvoir, malgré son habileté à déjouer les pièges qui guettent constamment celui qui mène un jeu complexe avec les médias, le censeur Rabkiewicz ne parvient pas à dissimuler sa profonde dépression. Ce quinquagénaire est un homme esseulé, délaissé par sa femme partie avec un homme plus jeune, et méprisé par sa fille qui a honte du métier de son père. Il souffre de façon évidente du syndrome de l'épuisement professionnel. Il a perdu toute foi dans le sens de son métier, ce qui influe sur son état de santé, car il souffre de problèmes cardiaques et de migraine chronique. Son état psychophysique n'est autre que le reflet du milieu dans lequel il évolue, mû par la force de l'habitude plutôt que par conviction. L'action du film se déroule dans une grande ville de la province polonaise, dans les années 1980, au moment du déclin de la République populaire de Pologne et après la fin de l'état de siège qui a étouffé Solidarność. La ville est laide : le long de ses rues étroites, on peut apercevoir des maisons négligées, des murs décrépits qui tombent en ruine. Tout annonce la décomposition en marche et la chute prochaine du régime. L'esprit de la décadence s'infiltre tant dans les mentalités que dans la matière. C'est le régime du marasme.

En outre, dans le travail routinier du censeur commencent à apparaître des situations auxquelles il ne parvient pas à faire face. Marczewski intensifie avec brio le sentiment d'absurdité grotesque en imprégnant l'univers de son film des symptômes d'un chaos impossible à maitriser. Le quotidien prend un cours de plus en plus irrationnel, ce qui met à l'épreuve les modes de pensée et les capacités cognitives du bon sens. Pour le censeur fatigué, le sentiment que son monde se désintègre, que la réalité qui l'entoure déraille et devient de plus en plus difficile à appréhender, est d'autant plus irritant que jusque-là il s'est toujours senti parfaitement à l'aise dans son monde et qu'il y a exercé le contrôle et le pouvoir. Désormais il se sent étranger dans un monde qui échappe non seulement à sa juridiction mais aussi à son entendement.

Cette dissonance dans l'appréhension de la réalité se manifeste d'abord à l'occasion de la dénonciation incompréhensible effectuée par les rédacteurs d'un journal contre eux-mêmes auprès du comité régional du parti. Leur journal a en effet publié un article sur la pollution de l'eau dans la ville, information qui normalement aurait dû être censurée. Cet acte de soumission grotesque met au jour le degré de terreur et de servilité qui caractérise l'attitude des milieux journalistiques vis-à-vis du pouvoir. La règle est de se protéger à tout prix. Lorsque le censeur, familier de ce genre d'intrigues, parvient à déchiffrer les mobiles complexes de cette dénonciation et trouve une façon de neutraliser ses adversaires, il apprend que les signataires ne sont pas membres du parti. Il est pris de panique parce qu'il pense que cette étrange 
dénonciation, d'une servilité répugnante, vise en fait les journalistes membres du parti. Cette situation alambiquée donne une bonne idée des méandres des relations avec le parti communiste, lui-même condamné à disparaitre parce que, pris dans ses propres contradictions et dans des manipulations bureaucratiques, il est incapable de résoudre les vrais problèmes de la société.

La dénonciation n'est qu'un prélude à la véritable révolte de la réalité à laquelle le censeur, cerné par l'absurde, doit faire face. En effet, on apprend par la suite que, dans un cinéma de la ville, les acteurs refusent de jouer dans le film qui est diffusé, ce qui semble amorcer une rébellion généralisée et nécessite une intervention rapide du censeur, responsable de l'orthodoxie idéologique des œuvres d'art. Cette idée de l'auteur du film a une dimension autoréflexive, parce qu' elle met en scène la réception du film et surtout parce qu'elle met en cause le principe de la mimesis réaliste en proposant un jeu original sur le sens métaphorique. Entreprise au nom de la liberté et de la dignité, la révolte des acteurs, dont les gestes et les mots ont pourtant été fixés pour toujours sur une bande enfermée dans une boîte en fer-blanc, acquiert une signification symbolique très forte. Marczewski a réussi à synthétiser dans cette métaphore la signification sociale et morale de la révolution de Solidarność, qui a commencé par les grèves des ouvriers des chantiers navals de Gdańsk en août I98I pour gagner rapidement des pans importants de la société polonaise. Le commencement de cette révolte se produit d'une façon inattendue : des individus (le directeur du cinéma, ancien agent des services secrets, d'abord, puis l'assistant du censeur) entonnent des fragments du Requiem de Mozart.

Les critiques ont attiré l'attention sur la dimension hypertextuelle de L'Évasion du cinéma "Liberté", qui se compose d'allusions à Crime et Châtiment de Dostö̈evski (le personnage de Raskolnikov), de citations de La Rose pourpre $d u$ Caire de Woody Allen, et surtout de clins d'œil insistants au Maître et Marguerite de Boulgakov : le prénom de la comédienne rebelle, Marguerite, qui inspire au censeur amoureux sa métamorphose spirituelle; la lune comme signe de la transcendance; l'hôpital psychiatrique où échoue le censeur épuisé par les manifestations surnaturelles; le chant mentionné ci-dessus qui rappelle la chorale des fonctionnaires de la Commission des spectacles et des délassements comiques, laquelle ne peut s'arrêter de chanter «Mer glorieuse, Baïkal sacré!» Le lien principal entre L'Évasion du cinéma "Liberté» et Le Maître et Marguerite n'a cependant jamais été relevé : c'est l'invasion à la fois destructrice et libératrice de la magie dans l'univers ordonné du totalitarisme où le parti communiste règne sans partage grâce aux deux piliers du régime répressif : la police politique et la censure. La troupe diabolique de Volland sème le désordre, le chaos et la désolation dans la capitale de l'empire, Moscou, dans les 
années I930, à l'apogée du stalinisme, ridiculisant les organes impuissants de la sécurité. De même, un demi-siècle plus tard, au moment du déclin de l'Empire soviétique, à la périphérie de celui-ci, dans une ville de province polonaise, un groupe de comédiens fait diversion, en propageant un ferment libéral, que ni la censure ni le pouvoir qui se tient derrière elle, ne peuvent maîtriser car ils sont impuissants face à l'irrationnel. L'écrivain russe qui avait écrit son roman pour se venger d'une série d'humiliations infligées par des apparatchiks staliniens semble avoir inspiré au réalisateur polonais le recours à une forme métaphorique pour montrer la mise en cause de l'ordre imposé par la force. Faisant appel aux forces irrationnelles, le roman comme le film parviennent, par le sarcasme, à désarmer et à rendre impuissant l'appareil de la terreur.

Marczewski pointe ironiquement les vains efforts des représentants du pouvoir dont le but est de tuer dans l'œuf la révolte des acteurs. Une cellule de crise est mise en place pour tenter d'empêcher la propagation du virus hors de l'enceinte du cinéma. Aux spectateurs du film qui, tout en se souvenant du massacre des ouvriers du littoral en décembre 1970, avaient, en septembre 1980, suivi avec angoisse l'affrontement entre l'appareil du pouvoir et les ouvriers grévistes des chantiers navals de Gdańsk, cette scène ne peut manquer de rappeler la menace mortelle d'alors. D'autant plus qu'y est exprimée l'exigence d'une solution radicale pour mettre un terme à une situation qui met le pouvoir en péril : la destruction de la copie du films. L'apparatchik qui propose cette solution met en garde : «Et si ça se propage à d'autres films et, Dieu nous en préserve, à la télévision, alors on ne pourra plus s'en sortir. [...] Ils pensent qu'ils sont libres. Personne ne pourra les retenir.»

L'idée originale de Marczewski, c'est la mise au jour de l'effacement des fonctions communicationnelles de la langue parlée par le pouvoir. Si ce dernier ne parvient pas à maîtriser la situation, c'est aussi parce qu'il ne peut pas la nommer. Il opère uniquement au moyen de lieux communs creux. Lorsque le censeur prend connaissance de ce qui se passe dans le cinéma, il a les mots suivants : "C'est une révolte de la matière puisque les hommes ont arrêté de se révolter." Bien que la situation soit à l'exact opposé de ce diagnostic, l'infantile assistant du censeur répète ce constat illusoire, vaine tentative de conjurer la réalité: "Tout est clair. C'est la matière qui se révolte.» Quand, à son tour, le censeur proteste contre la destruction de la pellicule et décide de rejoindre les rebelles, son supérieur, le secrétaire du comité du parti le met en garde : "Vous avez pris une décision politique!» Malgré sa foi dans la puissance du Verbe, son propos n'est qu'une gesticulation dénuée

5. Ceci fait référence à un fait bien connu dans le milieu du cinéma polonais : le projet de détruire la pellicule du film antistalinien L'Interrogatoire, de R. Bugajski, évoqué en réunion au début de l'état de siège, en avril 1982. 
de sens. De l'autre côté, cependant, la révolte réelle mais néanmoins complètement irrationnelle des acteurs acquiert peu à peu une légitimité totale, ce qui se reflète dans les réactions verbales des spectateurs de la séance interrompue, et se propage en écho dans la société. La question, apparemment anodine, de la dépendance au tabac du censeur en donne un bon exemple. Lorsque, bouleversé par le comportement des acteurs, le censeur allume une cigarette dans le cinéma, un des acteurs, l'instigateur de la révolte, lui intime, depuis l'écran, de l'éteindre. Interloqué, le censeur n'obtempère pas. C'est alors qu'un des spectateurs le rappelle à l'ordre et lance : "Écrase ton clope!» Plus tard, à l'hôpital psychiatrique, interrogé sur son état par le chef de service, le censeur rétorque : "Et vous, si un acteur à l'écran vous interdisait de fumer, comment vous sentiriez-vous?» Le chef de service ne partage pourtant pas le choc cognitif du censeur et demande : "Vous fumez beaucoup?" «Trente, quarante..." répond le censeur. "Alors il avait raison", constate le médecin, qui semble prendre l'étrange incident parfaitement au sérieux, sans faire attention aux circonstances extraordinaires, légitimant ainsi son sens. De cette manière, il participe lui aussi à la lente métamorphose du censeur.

Le point de non-retour dans le cheminement du censeur, qui à l'instar d'Alice aux pays des merveilles décide de passer de l'autre côté de l'écran et de se joindre à la révolte des acteurs ${ }^{6}$, correspond au refus de la destruction de la copie du film. Cette idée, digne de l'Inquisition, équivaut symboliquement à une exécution des grévistes du cinéma "Liberté», conformément à la logique du mélange entre réalité et fiction ${ }^{7}$. C'est le Rubicon moral que le censeur n'est pas en mesure de franchir : "J'estime qu'être une ordure c'est une chose, mais être un meurtrier c'en est une autre." Il retourne alors au cinéma, où, pour échapper aux apparatchiks qui le poursuivent, il franchit la frontière de l'écran qui symbolise le domaine de la liberté.

S'il se libère de l'emprise d'un pouvoir dépravé, il n'en est pas pour autant lavé des actes commis par le passé. Le censeur sera jugé sur les toits de la ville, où se tiennent les victimes de son activité professionnelle. L'accusation est représentée par le personnage de Raskolnikov qui pose à son bourreau d'autrefois la question essentielle : "Il y a eu crime et il devrait ne pas y avoir de châtiment?»

6. Cette trame fantastique a été directement inspirée à Marczewski par le film La Rose pourpre $d u$ Caire de Woody Allen, dont le héros, une star du cinéma, emprunte le chemin inverse, c'està-dire depuis l'écran vers la salle.

7. Cette propagation mène à un échange du statut ontique entre deux zones : lorsque le censeur apparaît à l'écran, il parle au secrétaire du parti figé dans une posture gombrowiczienne de la protestation : "Vous semblez tellement fictif.» 
L'Inventaire de Krzysztof Zanussi et L'Évasion du cinéma "Liberté» de Wojciech Marczewski mettent en scène des censeurs comme figures symboliques de la décomposition et de la chute du communisme en Pologne. Ces deux films soulèvent ainsi, au tournant des années 1980 et 1990, la question de la responsabilité morale du totalitarisme en Pologne. En 1989, à l'issue de la Table ronde, un consensus entre l'opposition incarnée par Solidarność et les communistes, qui ont accepté de céder le pouvoir pour ne pas avoir à payer leurs actes, a permis à la Pologne de recouvrer son indépendance politique. Mais les questions essentielles de la responsabilité de la violence et de l'étouffement de la liberté de penser ont été laissées en suspens pour préserver un compromis obtenu à grand-peine. Quinze ans plus tard, alors que la droite se trouvait au pouvoir, ce problème est revenu dans le débat public, mais il a perdu toute signification morale et a été instrumentalisé au profit de la politique et de la lutte pour le pouvoir. La leçon morale sur la lustration ${ }^{8}$ dispensée à la société polonaise par ces deux films n'a pas porté ses fruits.

8. La lustration est une mesure administrative utilisée par les régimes post-totalitaires pour exclure de la fonction publique les personnes ayant collaboré avec les services de sécurité communistes. 